\title{
Effects of fixed compared to automatic CPAP on sleep in Obstructive Sleep Apnoea Syndrome
}

\author{
O. Resta1 1 P. Carratù 1, A. Depalo1, T. Giliberti1, M. Ardito1, \\ O. Marrone2, G. Insalaco2
}

ABSTRACT: Effects of Fixed Compared to Automatic CPAP on Sleep in Obstructive Sleep Apnoea Syndrome. O. Resta, P. Carratù, A. Depalo, T. Giliberti, M. Ardito, O. Marrone, G. Insalaco.

Background: Automatic CPAP has been developed to improve CPAP efficiency and compliance. Continually matching the effective pressure may be associated to more frequent arousals that could disturb sleep. The aim of the present study was to compare sleep architecture after one month's home therapy with CPAP or with an AutoCPAP device.

Methods: Twenty OSAS patients (18 M / 2 F) after polysomnographic study with CPAP titration received either an automatic (AutoSet T, ResMed, Sydney, Australia) or a fixed level CPAP machine in a random, single blind fashion for one month. At the end of the home treatment period polysomnography was repeated while CPAP was administered by the same machine used at home.
Results: There was no significant difference between groups in terms of age (50.0 vs 45.5, NS), sex, BMI (38.3 vs 35.1, NS), RDI (45.4 vs 48.0, NS), and CPAP effective lev-

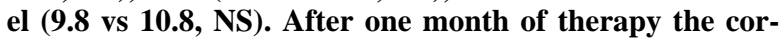
rection of sleep respiratory disturbances and of sleep structure was satisfactory in both groups. No difference in any polysomnographic variable or in subjective sleepiness was found at re-evaluation.

Conclusions: The results of this study demonstrate that on average CPAP administered by a fixed CPAP machine and by the AutoSet $T$ autoCPAP device has similar effects in improving respiratory function during sleep, nocturnal sleep architecture, and subjective daytime sleepiness after a one-month therapy. As autoCPAP devices are more expensive than fixed CPAP machines, their prescription should be considered only after a clear demonstration of an increase in compliance to treatment by these devices. Monaldi Arch Chest Dis 2004; 61: 3, 153-156.

Keywords: Sleep, obstructive sleep apnoea, ventilatory treatment.

1 Centro Universitario Disturbi Respiratori nel Sonno; Cattedra di Malattie dell'Apparato Respiratorio; Dipartimento di Metodologia Clinica e Tecnologie Medico - Chirurgiche; Facoltà di Medicina e Chirurgia - Università degli Studi di Bari. 2 Consiglio Nazionale delle Ricerche; Istituto di Biomedicina e Immunologia Molecolare "A. Monroy"; Sezione di Fisiopatologia Respiratoria - Palermo.

Correspondence: Prof. Onofrio Resta; Cattedra di Malattie dell'Apparato Respiratorio; Policlinico - P.zza Giulio Cesare, 11 70124 Bari; e-mail: oresta@pneumol.uniba.it

\section{Introduction}

Obstructive Sleep Apnoea Syndrome (OSAS) has an important social impact since it is associated with cardiovascular and neuropsychological disorders. OSAS is prevalent in $4 \%$ of middleaged men and $2 \%$ of middle-aged women [1]. Continuous Positive Airway Pressure (CPAP) is the most effectively established treatment. A major limitation to this therapy is compliance to treatment that can also be due to a poor tolerance of high pressure and to side effects.

Automatic CPAP has been developed to improve CPAP efficiency and compliance by automatically and continually matching the effective pressure to relieve upper airway obstruction. The effective pressure can change in relation to variations in body, jaw and neck position, alcohol ingestion, sleep stages [2-4], to long-term therapy itself or to changes in body weight [5]. Automatic CPAP ventilators administer a mean level of positive pressure during one night lower than fixed level CPAP machines [6,7]. This could lead to an improvement in compliance to treatment, as a lower pressure could be easier to apply and to tolerate, and high CPAP levels may be associated with more common side effects $[8,9]$. However, low-pressure levels may be associated with more frequent arousals that could disturb sleep and counterbalance the possible beneficial effect of a lower pressure, preventing the improvement in compliance. In a previous study we observed that low-pressure levels administered by an autoCPAP, applied for one night, could sometimes be responsible for arousals [10]. We are not aware if such effect may persist when autoCPAP is applied chronically.

The definition of effectiveness of home selfadjusting CPAP therapy during sleep is of major interest. The aim of the present study was to compare patients' sleep architecture, nocturnal respiratory function and daytime sleepiness improvement after a one-month's home therapy with fixed CPAP or with a ResMed Autoset T AutoCPAP device in two groups of subjects. 


\section{Patients and Methods}

Subjects with untreated sleep apnoea, referred to our sleep laboratory with clinical suspicion of OSAS, underwent a polysomnographic study. Their subjective sleepiness was evaluated by Epworth sleepiness scale (ESS). Polysomnographies were performed in our sleep laboratory. Signals were recorded by a computerised system (Compumedics Sleep Series W, Australia). They included: EEG (C4/A1, C3/A2), bilateral EOG, and chin EMG for sleep staging following criteria of Rechtschaffen and Kales [11], and for arousals scoring according to the American Sleep Disorders Associations criteria [12]; ECG; oro-nasal airflow detected by oro-nasal prongs connected to a pneumotachograph; thoracic and abdominal movements by inductive plethysmography; snoring by suprasternal microphone; oxyhemoglobin saturation by finger pulse oximeter (Palco Laboratory, model 305 AC). Apnoeas were defined as reductions in oro-nasal airflow of at least $90 \%$ for 10 seconds or more. Hyponoeas were defined as oronasal airflow reduction of at least $50 \%$ followed by a reduction in oxyhemoglobin saturation of $>$ $=4 \%$. Flow limitation events were defined as an airflow signal reduced by $<50 \%$ but flattened in its inspiratory branch for at least three consecutive breaths and followed by an arousal. The following parameters were measured: sleep efficiency (total sleep time as percentage of time spent in bed), stages 1, 2, 3-4 and REM as percentages of total sleep time, arousal index (ArI) (number of arousals per hour of sleep), respiratory disturbance index (RDI) (number of apnoeas, hyponoeas and flow limitation events per hour of sleep), snoring index (snores per hour of sleep), and minimum $\mathrm{SaO}_{2}$. Inclusion criteria were RDI $>30$ events/h of sleep with at least $80 \%$ of obstructive events, and indications for CPAP treatment according to the American Thoracic Society recommendations [13]. Patients with cardiac failure or lung disease were excluded. Twenty consecutive patients (18 male and 2 female) were recruited. Selected patients were middle-aged, obese; their RDI ranged between 30.7 and 79.7 events/hour.

CPAP titration was manually performed in the sleep laboratory during a polysomnographic study. Optimal CPAP was considered the lowest level of pressure that eliminated almost all obstructive respiratory events, including flow limitation events, in all body positions and in all sleep stages.

After titration, all subjects received either an automatic (AutoSet T, ResMed, Sydney, Australia) or a fixed level CPAP machine (Sullivan Elite, ResMed, Sydney, Australia) in a random, single blind fashion for one month. Fixed CPAP was set at the level determined during the titration study, while automatic CPAP was set so as to freely deliver pressure levels between 4 and $16 \mathrm{cmH}_{2} \mathrm{O}$. At the end of the home treatment period, data recorded by the machines was downloaded to evaluate their use; in addition pressure levels from autoCPAP machines were obtained, below which $>95 \%$ time was spent (95th centile CPAP). Then, polysomnography was repeated while CPAP was administered by the same machine used at home, and Epworth sleepiness score was re-evaluated.

Statistical analysis was performed using StatView 4.90 for windows (SAS Institute Inc., Cary, North Carolina, USA). Data is expressed as mean $\pm \mathrm{SD}$ values. Comparisons between variables recorded at the beginning and end of the study in each group were performed by paired Student's t test; comparisons between variables recorded in the two different groups were performed by unpaired Student's t test. ANOVA analysis was performed to evaluate differences between treatment effectiveness between groups. A $p$ value $<0.05$ was considered significant.

\section{Results}

All patients concluded the study. Age $(50.0 \pm 10.8$ vs 45.5 \pm 7.1 , fixed vs autoCPAP group) sex ( 9 male, 1 female), body mass index ( $38.3 \pm 7.2$ vs $35.1 \pm 7.1$ $\left.\mathrm{Kg} / \mathrm{m}^{2}\right)$, ESS $(12.0 \pm 3.2$ vs $15.7 \pm 5.1)$ did not differ between groups.

The first polysomnography showed less sleep at stage 1 and more sleep at stage 2 in the group of patients that underwent automatic CPAP compared to the fixed CPAP group (table 1). The manually titrated CPAP level did not differ between groups $(9.8 \pm 3.0$ vs $10.8 \pm 1.7$, fixed vs autoCPAP group, NS). Patients of the two groups used CPAP at home for a similar amount of time $(5.3 \pm 1.8$ and $5.2 \pm 1.4$ ) hours/day, respectively). Compliance to treatment was not related to severity of sleep respiratory disorders. No difference between groups in any polysomnographic variable (table 2 ) or in subjective sleepiness (ESS scores: $4.1 \pm 1.4$ and $5.2 \pm 2.9$ ), respectively) was found at re-evaluation. Patients belonging to both groups referred mild nasal symptoms. Correction of sleep respiratory disturbances and of sleep structure was satisfactory in both groups; differently from baseline studies, most residual events during CPAP polysomnographies were represented by flow limitation events. ANOVA analysis showed that CPAP treatment reduced significantly both RDI and ArI $(\mathrm{p}<0.001)$ without significant differences between groups. In the autoCPAP group, despite variable individual differences, no significant differences were observed among manually titrated CPAP level $(10.8 \pm 1.7)$, average 95th CPAP delivered in the month at home (9.8 \pm 1.4$)$, and $95^{\text {th }}$ CPAP level delivered during the last polysomnographic study $(10.1 \pm 1.3)$ by the same group. Manually titrated CPAP, and 95th CPAP obtained in the polysomnography night (figure 1 ), were significantly correlated $\left(\mathrm{r}^{2}=0.677, \mathrm{p}<0.005\right)$.

\section{Discussion}

Over the last ten years, automatic CPAP devices that allow self adjustments of pressure for sleep apnoea therapy have been developed $[9,14$, 15]. These devices are supposed to assure the lowest pressure necessary to keep upper airway patency. Such machines are based on different princi- 
Table 1. - Polysomnographic characteristics of patients at baseline in two groups

\begin{tabular}{lccc}
\hline & \multicolumn{2}{c}{ Faseline } & p \\
\hline TST (min) & $326.8 \pm 13.6$ & $362.55 \pm 53.1$ & $\mathrm{NS}$ \\
Sleep efficiency (\%) & $84.2 \pm 4.9$ & $86.9 \pm 8.0$ & $\mathrm{NS}$ \\
ArI & $43.1 \pm 9.1$ & $43.1 \pm 11.9$ & $\mathrm{NS}$ \\
Stage 1 (\%TST) & $20.5 \pm 6.8$ & $13.9 \pm 5.1$ & 0.025 \\
Stage 2 (\%TST) & $40.9 \pm 12.9$ & $51.3 \pm 8.5$ & 0.048 \\
Stage 3-4 (\%TST) & $22.8 \pm 12.5$ & $19.8 \pm 10.9$ & $\mathrm{NS}$ \\
Stage REM (\%TST) & $15.9 \pm 4.2$ & $15.0 \pm 8.1$ & $\mathrm{NS}$ \\
$\mathrm{RDI}$ & $45.3 \pm 10.7$ & $48.0 \pm 14.3$ & $\mathrm{NS}$ \\
Lowest $\mathrm{SaO}(\%)$ & $74.1 \pm 10.8$ & $72.4 \pm 10.5$ & $\mathrm{NS}$ \\
tSaO & $20.1 \pm 20.4$ & $38.0 \pm 28.1$ & $\mathrm{NS}$ \\
Snoring index & $259.8 \pm 98.1$ & $191.7 \pm 73.1$ & $\mathrm{NS}$ \\
\hline
\end{tabular}

TST: total sleep time; RDI: Respiratory Disturbance Index; ArI: Arousal Index; $\mathrm{tSaO}_{2}<90 \%$ : percentage of TST spent with a $\mathrm{SaO}_{2}<90 \%$.

Table 2. - Fixed CPAP vs Automatic CPAP after one month therapy

\begin{tabular}{lccc}
\hline & \multicolumn{2}{c}{ After one month therapy } & $\mathrm{p}$ \\
& Fixed & Auto & \\
\hline TST (min) & $331 \pm 28.7$ & $334.8 \pm 46.9$ & $\mathrm{NS}$ \\
Sleep efficiency (\%) & $86.2 \pm 7.3$ & $86.4 \pm 8.1$ & $\mathrm{NS}$ \\
ArI & $7.3 \pm 3.3$ & $7.4 \pm 2.3$ & $\mathrm{NS}$ \\
Stage 1 (\%TST) & $9.0 \pm 5.0$ & $10.6 \pm 10.7$ & $\mathrm{NS}$ \\
Stage 2 (\%TST) & $37.0 \pm 8.0$ & $33.8 \pm 14.0$ & $\mathrm{NS}$ \\
Stage 3-4 (\%TST) & $29.5 \pm 8.5$ & $33.8 \pm 11.0$ & $\mathrm{NS}$ \\
Stage REM $(\% \mathrm{TST})$ & $24.6 \pm 9.0$ & $21.7 \pm 12.4$ & $\mathrm{NS}$ \\
$\mathrm{RDI}$ & $8.4 \pm 3.6$ & $8.3 \pm 2.0$ & $\mathrm{NS}$ \\
Lowest $\mathrm{SaO}_{2}(\%)$ & $89.1 \pm 3.4$ & $88.3 \pm 3.3$ & $\mathrm{NS}$ \\
Snoring index & $15.9 \pm 9.0$ & $32.6 \pm 25.6$ & $\mathrm{NS}$ \\
$\mathrm{t} \mathrm{SaO}_{2}<90 \%(\%)$ & $0.3 \pm 0.5$ & $0.4 \pm 0.8$ & $\mathrm{NS}$ \\
\hline
\end{tabular}

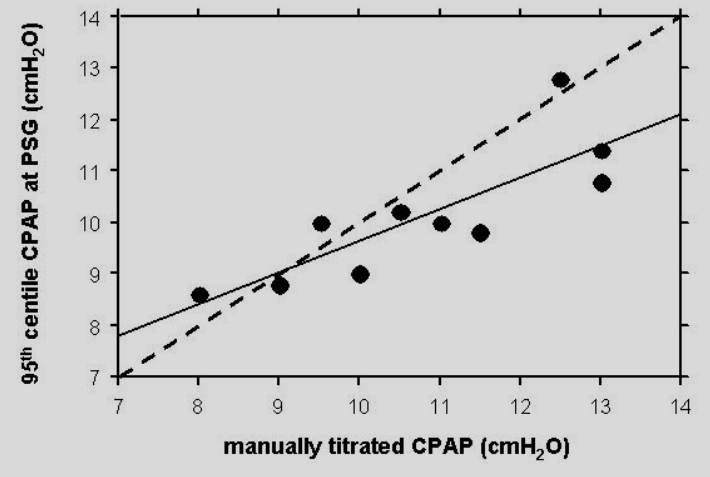

Fig. 1. - Correlation between manually titrated CPAP and $95^{\text {th }}$ centile CPAP recorded during polysomnography in the autoCPAP group. The dashed line is the line of identity.

ples of operation, so that theoretically each machine should be validated before use, as regards its effects both on breathing during sleep and on sleep structure. Such effects may be studied best after chronic application of the machines, when sleep structure is generally reverted to normal. In fact, in some patients the first application of a CPAP device is associated to a rebound effect on sleep structure, usually lasting few days, with extremely prolonged REM or slow wave sleep periods [16], and is immediately followed by a strong feeling of well-being; on the opposite, some patients require several nights of adaptation to the device before they are able to sleep continuously with the ventilator on, and they feel a benefit from its application. For these reasons we chose to compare the effects of fixed and of an automatic CPAP only after one month of nocturnal use.

The Autoset T device mainly operates modifying the pressure it administers after analysis of the flow wave profile, to prevent inspiratory flow limitation. The results of this study demonstrate that CPAP administered by a fixed CPAP machine and by the Autoset T autoCPAP device have similar effects in improving respiratory function during sleep, nocturnal sleep architecture and subjective daytime sleepiness after a one-month therapy. In these respects, these results do not differ from those of previous studies on different autoCPAP devices. In a chronic setting, irrespective of the machines under investigation, various studies reported that autoCPAP devices induce a similar improvement in sleep structure as fixed CPAP machines [14, 17-19].

In conclusion, the tested autoCPAP machines proved as effective as fixed CPAP in improving 
sleep quality, nocturnal respiratory disorders and subjective sleepiness. Therefore, in the average OSAS population, there is no advantage in the use of the AutoSet as compared to fixed CPAP. As they are more expensive than fixed CPAP, patients should be carefully selected before they are prescribed. Individual preference or a better compliance than to fixed CPAP could indicate the appropriateness of their prescription for home therapy in some patients.

\section{References}

1. Young T, Palta M, Dempsey J, et al. The occurrence of sleep disordered breathing among middle age adults. $N$ Engl J Med 1993; 328: 1230-1235.

2. Thut DC, Schwartz AR, Roach D, et al. Tracheal and neck position influence upper airway airflow dynamics by altering airway length. J Appl Physiol 1993; 75: 2084-2090.

3. Meurice JC, Marc I, Carrier G, et al. Effects of mouth opening on upper airway collapsibility in normal sleeping subjects. Am J Respir Crit Care Med 1996; 153: 255-259.

4. Sériès F, Marc I. Importance of sleep stage and body position-dependence of sleep apnoea in determining benefits to auto-CPAP therapy. Eur Respir J 2001; 18: 170-175.

5. Schwartz AR, Gold AR, Schubert N, et al. Effect of weight loss on upper airway collapsibility in obstructive sleep apnoea. Am J Respir Crit Care Med 1991; 144: 494-498.

6. Pilz K, Thalhofer S, Meissner P, et al. Improvement of CPAP therapy by a self-adjusting system. Sleep and Breathing 2000; 4: 169-172.

7. Teschler H, Berthon-Jones $\mathrm{M}$, Thompson $\mathrm{AB}$, et al. Automated continuous positive airway pressure titration for obstructive sleep apnea syndrome. Am J Respir Crit Care Med 1996; 154: 734-740.

8. Meslier N, Lebrun T, Grillier-Lanoir V, et al. A French survey of 3,225 patients treated with CPAP for obstructive sleep apnoea: benefits, tolerance, compliance and quality of life. Eur Respir J 1998; 12: 185-192.

9. Teschler H, Berthon-Jones M. Intelligent CPAP systems: clinical experience. Thorax 1998; 53: S49-54.

10. Marrone O, Insalaco G, Bonsignore MR, et al. Sleep structure correlates of CPAP variations during application of an autotitration CPAP machine in obstructive sleep apnea syndrome. Chest 2002; 121: 759-767.

11. Rechtschaffen A, Kales A (eds). A manual of standardized techniques and scoring system for sleep stages of human subjects. Los Angeles, Brain Info Service and Brain Res Inst, 1968.

12. Atlas task force of the American Sleep Disorders Association. EEG arousals: scoring rules and examples. Sleep 1992; 15: 174-184

13. Directors of ATS board. Indications and standards for use of nasal continuous airway pressure in sleep apnea syndromes. Am J Respir Crit Care Med 1994; 150: 1738-1745.

14. Meurice JC, Marc I, Sériès F. Efficacy of auto-CPAP in the treatment of obstructive sleep apnea/hypopnea syndrome. Am J Respir Crit Care Med 1996; 153: 794-798.

15. Lofaso F, Lorino AM, Duizabo D, et al. Evaluation of an auto-nCPAP device based on snoring detection. Eur Respir J 1996; 9: 1795-1800.

16. Issa FG, Sullivan CE. The immediate effects of nasal continuous positive airway pressure treatment on sleep pattern in patients with obstructive sleep apnea syndrome. Electroencephalogr Clin Neurophysiol 1986; 63: 10-17.

17. Konermann M, Sanner B, Vyleta M, et al. Use of conventional and self-adjusting nasal continuous positive airway pressure for treatment of severe obstructive sleep apnea syndrome: a comparative study. Chest 1998; 113: 714-718.

18. d'Ortho M-P, Grillier-Lanoir V, Lévy P, et al. Constant vs automatic continuous positive airway pressure therapy. Home evaluation. Chest 2000; 118: 1010-1017.

19. Teschler H, Wessendorf TE, Farhat AA, et al. Two months of autoadjusting versus conventional nCPAP for obstructive sleep apnoea syndrome. Eur Respir $J$ 2000; 15: 990-995.

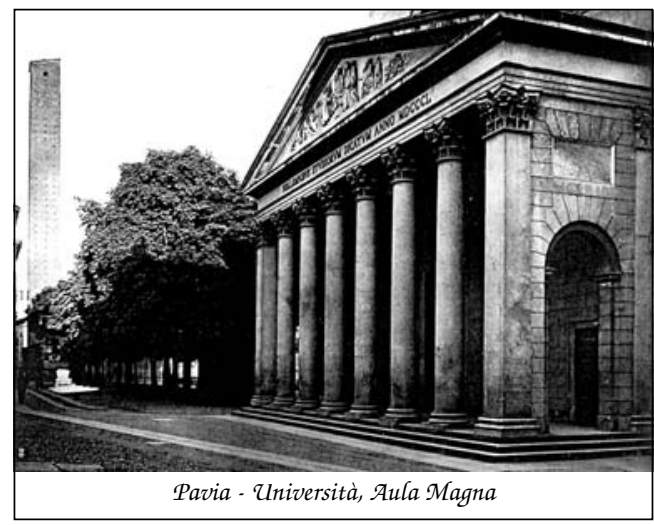

